\title{
Changes of abdominal muscle thickness during stable and unstable surface bridging exercise in young people
}

\author{
Tae Hoon Kim ${ }^{a}$, Joohee Hahn ${ }^{b}$, Ju-Ri Jeong ${ }^{c}$, Changjoo Lee ${ }^{d}$, You Jin Kim ${ }^{d}$, Sung Min Choi ${ }^{d}$, Da Young Jeon ${ }^{d}$, \\ Jin Hwa Lee ${ }^{d}$, In-Hyuk Lim ${ }^{e}$, Wan-Hee Lee ${ }^{d}$ \\ ${ }^{a}$ Sunshine Care Physical Therapy, New York, NY, USA \\ ${ }^{b}$ Department of Physical Therapy, The Graduate School, Sahmyook University, Seoul, Republic of Korea \\ ${ }^{c}$ Musculoskeletal Center, Samsung Seoul R\&D Medical Clinic, Seoul, Republic of Korea \\ dDepartment of Physical Therapy, College of Health and Welfare, Sahmyook University, Seoul, Republic of Korea \\ e Department of Physical Therapy, Yeoju Institute of Technology, Yeoju, Republic of Korea
}

Objective: The aim of this study was to measure the muscle architectural parameters of abdominal muscles in healthy individuals by rehabilitative ultrasound imaging (RUSI) and to investigate their changes after bridging exercise in various environments.

Design: Cross-sectional study.

Methods: The study included 40 healthy participants (19 men, 21 women). Subjects were randomly allocated to a stable surface group ( $S G, n=20)$ or an unstable surface group $(U G, n=20)$. The participants assumed three positions in rest, bridging exercise with knee flexion $60^{\circ}$, and bridging exercise with knee flexion $90^{\circ}$ for the measurement of abdominal muscle thickness by RUSI. For the resting position, the participants held the head neutral in a hook-lying position and the dominant side was measured. For contraction, the participants performed the bridging exercise with the knee joint in $60^{\circ}$ and $90^{\circ}$ of flexion for 10 seconds each.

Results: For transversus abdominis, external oblique muscle thickness, within the stable surface group and the unstable surface group, no significant contraction difference was observed in both the $60^{\circ}$ and $90^{\circ}$ bridge exercise conditions. Contraction difference of internal oblique muscle was significantly larger at $90^{\circ}$ than at $60^{\circ}$ within the $\mathrm{SG}(p<0.05)$. But within the UG, no significant contraction difference was shown. There was no significant contraction difference between the surface group and the unstable SG at $60^{\circ}$ condition and at $90^{\circ}$ condition in all measured muscles.

Conclusions: The contraction difference is different for each muscle during bridge exercise with knee flexion $60^{\circ}$ and bridging exercise with knee flexion $90^{\circ}$. Muscle contraction difference is generally large when exercised on an unstable surface than a stable surface, but these are not statistically significant when bridging exercise is performed using dynamic air cushion for unstable surface.

Key Words: Abdominal muscles, Abdominal oblique muscles, Exercise, Ultrasonography

\section{Introduction}

The core muscle, like the corset, is located in the lumbar-pelvic region [1]. The lumbar muscle is an important muscle because it provides mobility and stability during various movements [2]. The core stability is divided into the roles of global muscles and local muscles [3]. The local mus- cles are close to the spine and must act before the global muscle moves to stabilize the lumbopelvic region. On the other hand, the global muscles are located close to the surface and generate force and torque [1]. In order to stabilize the spine, it is important to harmonize the global and local muscles without activating any one muscle [4].

Bridging exercise is a typical method for stabilizing the

Received: 29 November, 2016 Revised: 16 December, 2016 Accepted: 19 December, 2016

Corresponding author: Wan-Hee Lee

Department of Physical Therapy, College of Health and Welfare, Sahmyook University, 815 Hwarang-ro, Nowon-gu, Seoul 01795, Republic of Korea Tel: 82-2-3399-1633 Fax: 82-2-3399-1639 E-mail: whlee@syu.ac.kr

(c) This is an Open-Access article distributed under the terms of the Creative Commons Attribution Non-Commercial License (http://creativecommons.org/licens es/by-nc/4.0) which permits unrestricted non-commercial use, distribution, and reproduction in any medium, provided the original work is properly cited.

Copyright $\odot 2016$ Korean Academy of Physical Therapy Rehabilitation Science 
trunk muscles. It is known to enhance muscle coordination patterns to generate the overall torque of the global muscle and the stability of the local muscle [4]. Bridging exercise enhance the neuromuscular control of trunk flexor and extensor muscles and enhancing stability of the trunk and lumbar region by strengthening the pelvis and lower limb muscles [5]. In addition, many studies have been conducted through various methods and modifications. To evaluate the effects of bridging exercise, electromyography is often used [1,5-9]. However there is still a lack of study to investigate the mechanism of motor function improvement after bridging exercise, especially from the muscle fascicle level.

The muscle architecture defined by the geometric arrangement of the fascicle affects muscle function which characterizes and specifies the muscle's ability to generate power [10]. Recently, rehabilitative ultrasound imaging (RUSI) has been applied to measure skeletal muscle structure $[11,12]$. RUSI can distinguish between muscle and tissue to produce high-quality images of the muscle structure. It is also possible to quantitatively measure muscle structure such as fiber size, thickness, length, cross-sectional area, and pennation angle [13].

The purpose of this study was, therefore, to measure the muscle architectural parameters of transversus abdominis ( $\operatorname{TrA}$ ), internal oblique (IO), and external oblique (EO) in healthy individuals by RUSI and to investigate their changes after bridging exercise in various environments. It will help us to understand the biomechanical mechanisms of exercise. We hypothesize that RUSI could distinguish the changes after bridging exercise and that the exercise might be related to the changes of muscle architecture.

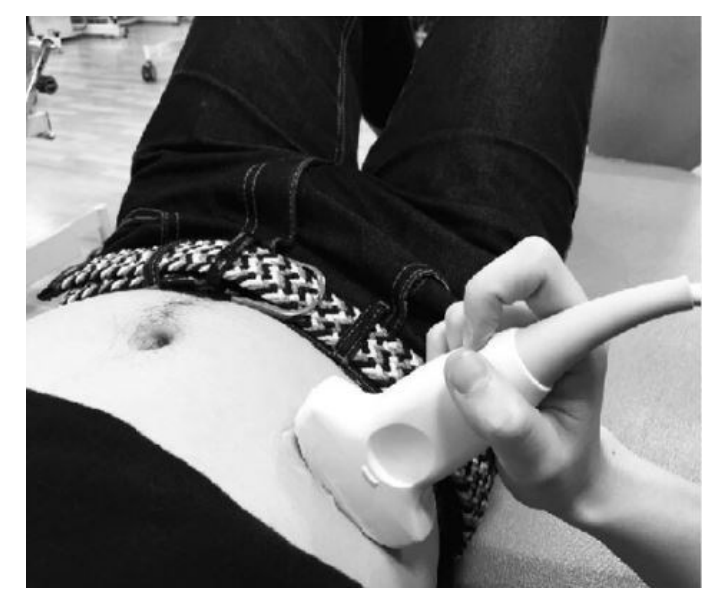

Figure 1. Placement of probe.

\section{Methods}

\section{Subjects}

Forty healthy individuals (19 men, 21 women) voluntarily participated in the study. Healthy subjects aged between 18 and 28 years, with a full active range of motion and who had no history of skeletal muscle pain were recruited for the study. The exclusion criteria were: past or present musculoskeletal or neuromuscular disorders in the lower extremity, pelvic, and lower back; pregnancy; malignant tumors; and obesity body mass index $(\mathrm{BMI})>30 \mathrm{~kg} / \mathrm{m}^{2}$. The study was approved by the Institutional Review Board of the Sahmyook University (SYUIRB 2-1040781-AB-N-012016110HR) in Seoul.

\section{Procedures}

Subjects were randomly allocated to a stable surface group ( $\mathrm{SG}, \mathrm{n}=20)$ or an unstable surface group (UG, $\mathrm{n}=20)$. SG performed these exercise on the ground and UG put a dynamic air cushion (TOGU, Prien-Bachham, Germany) under the feet. The participants assumed three positions at rest, bridging exercise with knee flexion $60^{\circ}$ (BEKS), and bridging exercise with knee flexion $90^{\circ}(\mathrm{BEKN})$ for the measurement of abdominal muscle thickness. For the resting position, the participants held the head neutral in a hook-lying position; the dominant side was measured. For contraction, the participants performed the bridging exercise with the knee joint at $60^{\circ}$ flexion for 10 seconds, and afterward with the knee joint flexion $90^{\circ}$.

Muscle thickness measurement was performed with a Medison Mysono portable ultrasound system (U5; Samsung Medison, Seoul, Korea). In this study, the portable ultra-

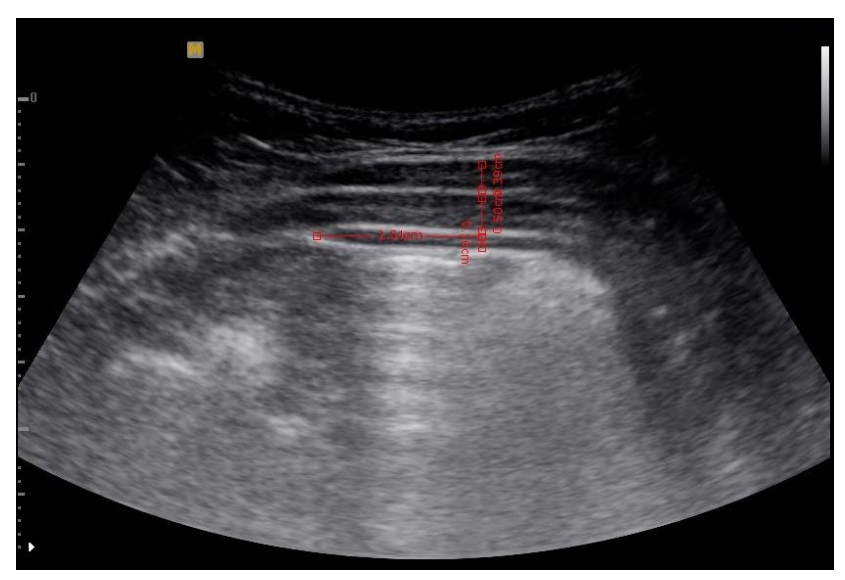

Figure 2. Measurement of muscle thickness. 
sound with a 5-MHz curvilinear transducer was used to obtain images [14]. The probe was placed horizontally on the middle of abdominal region between the 11th costal cartilage and the anterior superior iliac crest (Figure 1) [15]. A coupling gel was applied to contact between the ultrasound probe and skin surface of the subject. At the end of the contraction, the image was captured and saved.

We analyzed the image and calculated measurements of the abdominal muscle using Sante DICOM Viewer (Santesoft Ltd., Athens, Greece). The image was measured along a horizontal reference line located $2.5 \mathrm{~cm}$ from the edge muscle-fascia junction of TrA. Then the thickness of three muscles layers (EO, IO, TrA) were measured by drawing a vertical line in the horizontal reference line (Figure 2).

\section{Statistical analysis}

The data were analyzed by PASW Statistics ver. 18.0 (IBM Co., Armonk, NY, USA). Comparisons of subject'

Table 1. Characteristics of the subjects

$(\mathrm{N}=40)$

\begin{tabular}{lcc}
\hline \multicolumn{1}{c}{ Parameter } & $\mathrm{SG}(\mathrm{n}=20)$ & $\mathrm{UG}(\mathrm{n}=20)$ \\
\hline Gender (male/female) & $9 / 11$ & $10 / 10$ \\
Age $(\mathrm{y})$ & $22.65(1.87)$ & $22.90(1.97)$ \\
Height $(\mathrm{cm})$ & $166.92(5.98)$ & $168.51(9.18)$ \\
Weight $(\mathrm{kg})$ & $60.95(7.87)$ & $59.57(11.96)$ \\
Body mass index $\left(\mathrm{kg} / \mathrm{m}^{2}\right)$ & $21.79(1.67)$ & $20.76(2.42)$ \\
Waist $(\mathrm{cm})$ & $78.17(6.49)$ & $76.84(9.20)$ \\
\hline
\end{tabular}

Values are presented as number only or mean (SD).

SG: stable surface group, UG: unstable surface group.

Table 2. Comparison of baseline muscle thickness $\quad(\mathrm{N}=40)$

\begin{tabular}{llccc}
\hline Muscle & $\begin{array}{c}\text { Condition } \\
(\mathrm{mm})\end{array}$ & $\begin{array}{c}\text { SG } \\
(\mathrm{n}=20)\end{array}$ & $\begin{array}{c}\mathrm{UG} \\
(\mathrm{n}=20)\end{array}$ & $\mathrm{t}(p)$ \\
\hline TrA & Rest & $2.55(0.72)$ & $2.46(0.81)$ & $0.358(0.722)^{\mathrm{a}}$ \\
& BEKS & $3.54(1.25)$ & $3.57(1.52)$ & \\
& BEKN & $4.02(1.47)$ & $3.68(1.60)$ & \\
IO & Rest & $7.78(1.56)$ & $6.78(2.12)$ & $1.689(0.099)$ \\
& BEKS & $8.07(2.05)$ & $8.16(2.85)$ & \\
& BEKN & $8.65(2.04)$ & $8.05(2.63)$ & \\
EO & Rest & $5.05(1.06)$ & $4.43(1.12)$ & $1.772(0.084)$ \\
& BEKS & $4.69(1.35)$ & $3.87(1.07)$ & \\
& BEKN & $4.76(1.03)$ & $4.07(1.21)$ & \\
\hline
\end{tabular}

Values are presented as mean (SD).

SG: stable surface group, UG: unstable surface group, TrA: transversus abdominis, IO: internal oblique, EO: external oblique, BEKS: bridging exercise with knee flexion $60^{\circ}$, BEKN: bridging exercise with knee flexion $90^{\circ}$.

${ }^{\text {a }}$ Comparison of baseline muscle thickness in rest condition general characteristics were performed using the independent t-test. Rest, BEKS, and BEKN data were analyzed using the paired t-test to describe within-group differences. The independent t-test to test differences between the groups. The statistical significance level was 0.05 for all analyses.

\section{Results}

\section{Demographic characteristics}

The participants' characteristics are presented in Table 1. No significant differences in general characteristics were observed between the SG and the UG. 40 healthy participants (male $=19$, female $=21$ ) with mean age 22.65 and 22.90 years, mean weight 60.95 and $59.57 \mathrm{~kg}$, mean height 166.92 and $168.51 \mathrm{~cm}$, mean BMI 21.79 and $20.76 \mathrm{~kg} / \mathrm{m}^{2}$, and waist circumference 78.17 and $76.84 \mathrm{~cm}$, respectively.

\section{Comparison of abdominal muscles (TrA, IO, EO) thick- ness}

A summary of the results for the change of TrA muscle thickness is shown in Table 2 and 3. For TrA muscle thickness, within the $\mathrm{SG}$, no significant contraction difference was observed in both the $60^{\circ}$ and $90^{\circ}$ conditions. The same result in the UG was observed. There was no significant contraction difference between the surface group and the unstable stable surface group at $60^{\circ}$ condition. The same result was obtained at the $90^{\circ}$ condition.

The thickness variation of IO is shown in Table 2 and 3.

Table 3. Comparisons of muscle contraction difference $(\mathrm{N}=40)$

\begin{tabular}{lcccr}
\hline Muscle & $\begin{array}{c}\text { Condition } \\
(\mathrm{mm})\end{array}$ & $\begin{array}{c}\mathrm{SG} \\
(\mathrm{n}=20)\end{array}$ & $\begin{array}{c}\mathrm{UG} \\
(\mathrm{n}=20)\end{array}$ & $\mathrm{t}(p)$ \\
\hline \multirow{2}{*}{ TrA } & $\mathrm{SR}$ & $0.98(1.35)$ & $1.10(1.74)$ & $0.238(0.813)$ \\
& $\mathrm{NR}$ & $1.47(2.17)$ & $1.21(1.82)$ & $-0.402(0.690)$ \\
& $\mathrm{t}(p)$ & $1.830(0.083)$ & $0.479(0.637)$ & \\
$\mathrm{IO}$ & $\mathrm{SR}$ & $0.29(2.16)$ & $1.37(2.66)$ & $1.416(0.165)$ \\
& $\mathrm{NR}$ & $0.87(2.15)$ & $1.27(2.08)$ & $0.600(0.552)$ \\
& $\mathrm{t}(p)$ & $2.489(0.022)^{*}$ & $0.459(0.652)$ & \\
EO & $\mathrm{SR}$ & $0.36(1.07)$ & $0.56(0.96)$ & $-0.620(0.539)$ \\
& $\mathrm{NR}$ & $0.28(1.01)$ & $0.36(0.78)$ & $-0.287(0.776)$ \\
& $\mathrm{t}(p)$ & $0.558(0.583)$ & $1.147(0.265)$ & \\
\hline
\end{tabular}

Values are presented as mean (SD).

SG: stable surface group, UG: unstable surface group, TrA: transversus abdominis, IO: internal oblique, EO: external oblique, SR: contraction difference of rest and bridging exercise with knee flexion $60^{\circ}$, NR: difference in contraction of rest and bridging exercise with knee flexion $90^{\circ}$.

${ }^{*} p<0.05$. 
For the $\mathrm{SG}$, the contraction difference was significantly larger at $90^{\circ}$ than at $60^{\circ}$. But for the UG, no significant contraction difference was shown in both the $60^{\circ}$ and $90^{\circ}$ conditions. There was no significant difference between groups in both $60^{\circ}$ and $90^{\circ}$ conditions. The results of EO are shown in Table 2 and 3, and are not different from those of TrA.

\section{Discussion}

Stabilization of the trunk is necessary to improve the stability of the spine and pelvis in functional postures and movements, to strengthen the involved muscles, and to regulate and balance muscle and movement [16]. Bridging exercises for the stabilization of lumbopelvic focuses on retraining the coordination patterns of muscles between stabilization of local muscles and torque formation of global muscles [7].

The aim of this study was to measure the muscle architectural parameters of abdominal muscles in healthy individuals by RUSI and to investigate their changes after bridging exercise in various environments.

The results of the present study correspond well with those found in the earlier experimental studies. Lee et al. [8], reported that the the muscle activity of EO was higher than that of knee flexion $90^{\circ}$ at the knee angle $60^{\circ}$ but not significant. In this study, the contraction difference of EO was greater at $60^{\circ}$ than at $90^{\circ}$ but not statistically significant in both stable and UGs. In a previous study, when the knee angle was $90^{\circ}$ and $60^{\circ}$ in the bridging exercise, muscle activity was higher at $60^{\circ}$ than at $90^{\circ}$ for all the muscles measured. However, in gluteus medius, it was higher at $90^{\circ}$ than at $60^{\circ}$ [8]. In this study, the contraction difference of $\operatorname{TrA}$ was larger at $90^{\circ}$ than at $60^{\circ}$. The contraction difference of IO was significantly larger at $90^{\circ}$ than at $60^{\circ}$ in SG. This result shows that the contraction or activity of the muscles according to the angle of the knee during bridging exercise may be different depending on the muscles.

Czaprowski et al. [3] measured the muscle activity of rectus abdominis (RA), EO, and IO-TrA by surface electromyography (EMG) while performing bridging exercises in various positions. There was no significant difference in muscle activity between supine bridge on stable surface and supine bridge on a BOSU; a balance cushion similar to TOGU. In the present study, there was no statistically significant difference in muscle contraction between the stable and unstable groups, resembling the results in the previous research.
In this study, although not statistically significant, the muscle contraction difference in the UG is larger than that of the SG at the same knee joint angle of IO and EO at the $60^{\circ}$ knee joint condition of the TrA. In previous studies, the maximum voluntary isometric contraction (MVIC) values of the muscles were measured by EMG when performing normal bridging exercise (NBE), bridging with ball exercise (BBE), and bridging with sling exercise (BSE). As a result, \%MVIC values were significantly higher during $\mathrm{BSE}$ than during BBE and NBE in IO, RA, multifidus, and erector spinae (ES). Especially in RA and ES, \%MVIC values were significantly higher during BBE than during NBE [9]. In another previous study, normal back bridging (NB), back bridging using swiss ball (BUB) and back bridging using sling (BUS) are performed. Activation of TrA during BUB was significantly higher than during NB and BUS [1]. These results are similar to our studies.

The conclusion of this study is that the contraction difference according to the knee angle is different for each muscle during bridging exercise. Muscle contraction difference is generally large when exercised on an unstable surface than a stable surface, but these are not statistically significant when bridging exercise is performed using TOGU with an unstable surface.

This study still has limitations to be discussed about using ultrasound to assess muscle structure. The EMG application method according to the change of the bridging posture is specified in the previous study, but there is no agreement about the RUSI yet. Finally, the number of subjects was limited. Future studies will require a larger sample size or multi-center design with a more specific protocol.

\section{Conflict of Interest}

The authors declared no potential conflicts of interest with respect to the authorship and/or publication of this article.

\section{References}

1. Ahmad I, Sharma S. Electromyographic activity profile of transverse abdominis and multifidus muscles during bridging exercise variants in colligiate cricketers. Physiother Occup Ther 2014;7:161-71.

2. Nabavi N, Mosallanezhad Z, Haghighatkhah HR, Mohseni Bandpeid MA. Reliability of rehabilitative ultrasonography to measure transverse abdominis and multifidus muscle dimensions. Iran J Radiol 2014;11:e21008. 
3. Czaprowski D, Afeltowicz A, Gębicka A, Pawłowska P, Kędra A, Barrios C, et al. Abdominal muscle EMG-activity during bridge exercises on stable and unstable surfaces. Phys Ther Sport 2014; 15:162-8.

4. Stevens VK, Coorevits PL, Bouche KG, Mahieu NN, Vanderstraeten GG, Danneels LA. The influence of specific training on trunk muscle recruitment patterns in healthy subjects during stabilization exercises. Man Ther 2007;12:271-9.

5. Park HJ, Oh DW, Kim SY. Effects of integrating hip movements into bridge exercises on electromyographic activities of selected trunk muscles in healthy individuals. Man Ther 2014;19:246-51.

6. Feldwieser FM, Sheeran L, Meana-Esteban A, Sparkes V. Electromyographic analysis of trunk-muscle activity during stable, unstable and unilateral bridging exercises in healthy individuals. Eur Spine J 2012;21 Suppl 2:S171-86.

7. Stevens VK, Bouche KG, Mahieu NN, Coorevits PL, Vanderstraeten GG, Danneels LA. Trunk muscle activity in healthy subjects during bridging stabilization exercises. BMC Musculoskelet Disord 2006;7:75.

8. Lee SK, Moon DC, Cho HR, Kim TY. Effects of trunk and neck extensor muscle activity on the bridging exercise according to knee joint angle. J Phys Ther Sci 2013;25:363-5.

9. Kang H, Jung J, Yu J. Comparison of trunk muscle activity during bridging exercises using a sling in patients with low back pain. J Sports Sci Med 2012;11:510-5.

10. Liu P, Wang Y, Hu H, Mao Y, Huang D, Li L. Change of muscle architecture following body weight support treadmill training for persons after subacute stroke: evidence from ultrasonography. Biomed Res Int 2014;2014:270676.

11. Kim MK, Ko YJ, Lee HJ, Ha HG, Lee WH. Ultrasound imaging for age-related differences of lower extremity muscle architecture. Phys Ther Rehabil Sci 2015;4:38-43.

12. Lee KB, Kim JG, Park HG, Kim JE, Kim HS, Lee WH. Correlation between lateral abdominal, rectus femoris, and triceps brachii muscle thickness and endurance during prone bridge exercise in healthy young adults. Phys Ther Rehabil Sci 2015;4:11-6.

13. Jeong JR, Han JH, Cho JE, Lee WH. Reliability and validity of a personal computer based muscle viwer for measuring upper trapezius and transveres abdominis muscle thickness. Phys Ther Rehabil Sci 2016;5:155-61.

14. Koppenhaver SL, Parent EC, Teyhen DS, Hebert JJ, Fritz JM. The effect of averaging multiple trials on measurement error during ultrasound imaging of transversus abdominis and lumbar multifidus muscles in individuals with low back pain. J Orthop Sports Phys Ther 2009;39:604-11.

15. Ko YJ, Ha HG, Jeong J, Lee WH. Variations in lateral abdominal muscle thickness during abdominal drawing-in maneuver in three positions in a young healthy population. Phys Ther Rehabil Sci 2014;3:101-6.

16. Richardson CA, Snijders CJ, Hides JA, Damen L, Pas MS, Storm $\mathrm{J}$. The relation between the transversus abdominis muscles, sacroiliac joint mechanics, and low back pain. Spine (Phila Pa 1976) 2002;27:399-405. 